\title{
The Frequency of Resurgery after Percutaneous Lumbar Surgery Using Dekompressor in a Ten-Year Period
}

\author{
Stephan Klessinger (iD) ${ }^{1,2}$ \\ ${ }^{1}$ Department of Neurosurgery, Nova Clinic Biberach, Eichendorffweg 5, 88400 Biberach, Germany \\ ${ }^{2}$ Department of Neurosurgery, University of Ulm, Albert-Einstein-Allee 23, $89081 \mathrm{Ulm}$, Germany \\ Correspondence should be addressed to Stephan Klessinger; klessinger@nova-clinic.de
}

Received 3 May 2018; Accepted 25 September 2018; Published 9 October 2018

Academic Editor: Peng Hui Wang

Copyright (c) 2018 Stephan Klessinger. This is an open access article distributed under the Creative Commons Attribution License, which permits unrestricted use, distribution, and reproduction in any medium, provided the original work is properly cited.

\begin{abstract}
To prevent open surgical procedures, minimally invasive techniques, like Dekompressor (PLDD), have been developed. The absence of reherniation is an important factor correlating with clinical success after lumbar surgery. In this retrospective, observational study, the frequency of additional open surgery after PLDD in a long time retrospective was examined. The correlation between clinical symptoms and outcome was assessed, and the time between PLDD and open surgery was analyzed. Consecutive patients after PLDD between 2005 and 2007 were included. MacNab's outcome criteria were used to evaluate patient satisfaction. The need for additional open surgery of the lumbar spine, the period between Dekompressor and resurgery, and the treated levels were analyzed. In total, 73 patients were included in this study. The patients were seen one month after PLDD. The majority of patients (76.7\%) had additional radicular pain. The most common level treated was L4-5 (58.9\%). The follow-up time was longer than 5 years in $30.1 \%$ of the patients and longer than 10 years in $6.82 \%$. The short-term success rate was $67.1 \%$. Additional surgery was performed in $26.0 \%$ of patients, with $78.9 \%$ of the reoperations undertaken during the first year after PLDD. These patients had a statistically significant worse outcome $(\mathrm{P}=0.025)$. Radicular pain was present in all patients with an early subsequent surgery, but only in $50 \%$ of patients with late surgery $(\mathrm{P}=0.035)$. Significantly more patients with poor pain relief had radicular pain $(\mathrm{P}=0.04)$. The short-term success rate was worsened by a resurgery rate of $26.0 \%$. Subsequent surgery, a short time after PLDD, suggests that PLDD is not a replacement for open discectomy. Because patients with radicular pain had a worse outcome and more frequent resurgeries, whether radicular pain is an ideal indication for PLDD should be discussed.
\end{abstract}

\section{Introduction}

Lumbar radicular pain caused by disc herniation is often treated with open discectomy [1]. Its effectiveness has been demonstrated in controlled trials [2-4] and in long-term follow-up studies [5]. Minimally invasive techniques have been developed to prevent open surgery. The paucity of evidence supporting these minimally invasive techniques highlights the need for more data. Only limited evidence exists for Nucleoplasty and Dekompressor [6,7].

Percutaneous lumbar disc decompression with Dekompressor (PLDD) uses the Archimedes' pump principle to mechanically remove a predetermined amount of disc material, reducing the pressure in the disc. The placement of the 1.5 $\mathrm{mm}$ cannula is similar to that used in a standard discography.

PLDD has been shown to be superior to conservative treatment $[6,8,9]$ and has been associated with a low rate of complications [8]. However, limited outcome data are available. While preliminary studies revealed a favorable outcome [9-13], only two assessed clinical outcomes beyond one year $[6,10]$, and only one study measured the open surgery rate after PLDD [6]. The absence of reherniation is an important factor for patient satisfaction after discectomy [14]. The expected outcomes after revision surgery are less well defined than for primary discectomy [14]. Therefore, the number of subsequent surgeries after PLDD is of great importance.

It is important to find an ideal indication for PLDD. Unsuccessful conservative treatment is a prerequisite of any spine surgery. Patients with a clear indication for open discectomy are also not ideal candidates for a percutaneous technique. It seems that outcomes after microdiscectomy for contained herniations are worse than for sequestered herniations [15]. As such, Dekompressor has been devised 


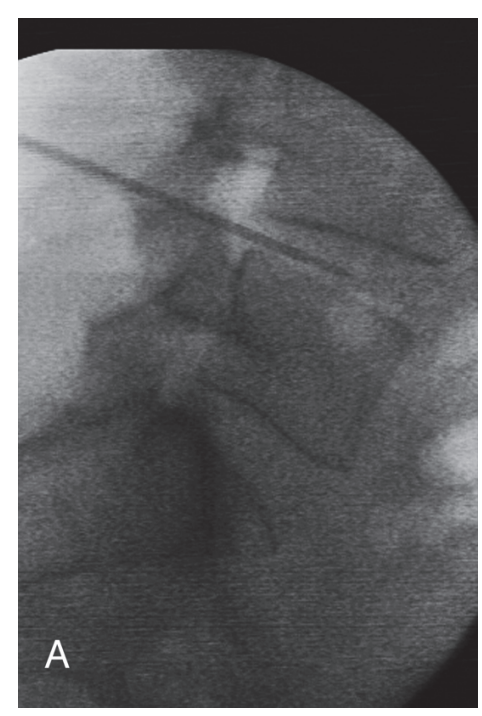

(a)

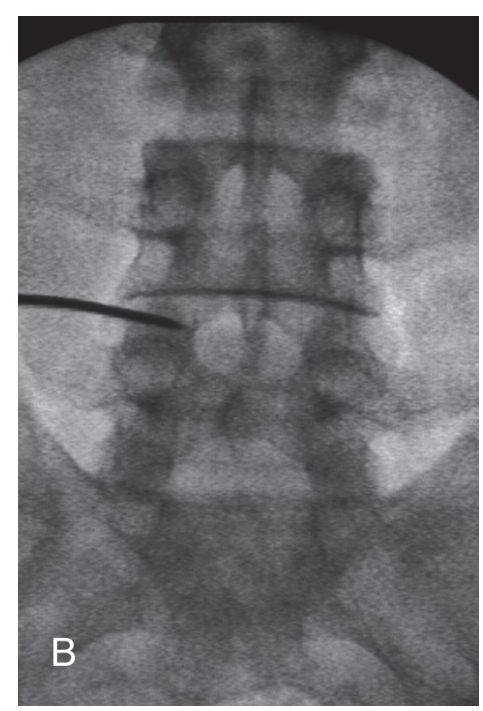

(b)

FIGURE 1: AP and lateral fluoroscopy image of the position of the PLDD wand at L4-5.

for small contained disc herniations [1]. The idea is that the nucleus and annulus are in a closed system where the herniated part can move back towards the center after a decrease in volume. A contained disc is regarded as an important prerequisite to the success of PLDD [8].

Advantages of the Dekompressor system are the minimal damage to adjacent tissues [11]. Proponents of the system state that Dekompressor does not substitute disc degeneration [11]. Less pertinent scarring and less postoperative fibrosis may be expected [12].

The aim of this retrospective, observational study was to investigate the frequency of an additional open surgery after PLDD in a more than ten-year retrospective. The time between PLDD and open surgery was analyzed, and a correlation between the clinical symptoms and outcome was assessed.

\section{Materials and Methods}

In this retrospective observational study, the patient data were drawn from an electronic medical record system. PLDD was performed in a practice setting. Open disc surgery was performed in a general hospital.

Inclusion criteria were as follows: consecutive patients who underwent PLDD between January 2005 and December 2007. A history of pain for a minimum of 3 months was mandatory. Patients had either low back pain or radicular pain with or without a sensory loss. Patients with a lumbar spine surgery in their history were excluded.

For PLDD, the 17-gauge Dekompressor probe (Stryker, Kalamazoo, Michigan) was used. Prophylactic antibiotics were administered prior to the procedure. A standard approach was used to place the introducer cannula with the stylet under fluoroscopic view intradiscally. The correct cannula placement was confirmed with anterior-posterior and lateral fluoroscopic images (Figure 1). The Dekompressor probe was advanced into the cannula and switched on. Disc material was harvested by moving the cannula along several passes intradiscally.

Every patient was seen in the practice personally one month after the operation for follow-up and later according to the complaints of the patient. A physician interview and a clinical examination were performed. A long-term follow-up of more than ten years was possible.

The age and gender of the patients, the treated levels, the follow-up time, and the pain characteristics (only lumbago or radicular pain with or without sensory loss) were evaluated. MacNab's outcome criteria [14] ( 1 = Excellent, no pain, no restriction of activity; 2 = Good, occasional pain; 3 = Fair, improved but handicapped by intermittent pain; $4=$ Poor, no improvement) were used to measure the success after PLDD. The evaluation of the necessity of an additional open lumbar spine surgery was the focus of this study. The period of time between the PLDD and the resurgery and the treated levels and the symptoms of the patients (back pain or radicular complaints) were analyzed.

The Exact-Fisher-test was used to compare values of patients with substantial pain relief and poor pain relief. Welch's t-test was used to test the hypothesis that two populations had equal means. $\mathrm{P}<0.05$ was set as the threshold for interpreting the results as significant.

\section{Results}

Between January 2005 and December 2007, 86 patients were treated with PLDD. Because of spine surgery in their history, eleven patients were excluded. Two patients were lost to follow-up. Therefore, 73 patients were included in this study. The data of these patients are shown in Table 1. In total, 33 patients (45.2\%) were women and 40 were men. The age of the patients was between 17 and 85 years, with the mean age being 48.9 years. 
TABLE 1: Patient characteristics.

\begin{tabular}{lc}
\hline \multicolumn{2}{c}{ All Patients } \\
\hline $\mathrm{n}$ & 73 \\
\hline Age (years) & \\
$\quad$ mean & $48.9 \pm 13.4$ \\
$\quad$ min-max & $17-85$ \\
Female & $33(45.2 \%)$ \\
Level & \\
L2-3 & $2(2.7 \%)$ \\
L3-4 & $4(5.5 \%)$ \\
L3-4-5 & $3(4.1 \%)$ \\
L4-5 & $43(58.9 \%)$ \\
L4-5-S1 & $8(11.0 \%)$ \\
L5-S1 & $13(17.8 \%)$ \\
Side & \\
left & $37(50.7 \%)$ \\
$\quad$ right & $31(42.5 \%)$ \\
$\quad$ both & $5(6.8 \%)$ \\
Radicular Pain & $56(76.7 \%)$ \\
Sensory Loss & $43(58.9 \%)$ \\
Follow-up (months) & \\
mean & \\
min-max & $35.6 \pm 40.2$ \\
Macnab's outcome criteria & $1-132$ \\
mean & \\
substantial pain relief (1+2) & $2.2 \pm 1.0$ \\
Pdditional surgery at index level & $49(67.1 \%)$ \\
mean & $19(26.0 \%)$ \\
$\quad$ min-max & \\
\hline & $10.1 \pm 17.1$ \\
& $1-70$ \\
\hline
\end{tabular}

All patients had pain for more than three months (mean 6.6 months). Twenty-eight patients (38.4\%) reported pain for more than one year before the treatment. Seventeen patients only had back pain. The majority of the patients $(76.7 \%)$ had additional radicular pain. A sensory loss in the symptomatic leg was present in 43 patients (58.9\%). No motor deficit was present.

The most common level treated was L4-5 (58.9\%). Two levels (either L3-4-5 or L4-5-S1) were treated in 11 patients (15.1\%). In $50.7 \%$ of patients the left side was symptomatic, while in $42.5 \%$ of the patients, the right side was treated. Five patients $(6.8 \%)$ were treated on both sides. No PLDD-related severe complications occurred.

The first follow-up examination one month after PLDD was mandatory for all patients. Further examinations were arranged according to the needs of the patients. This first follow-up was the only one in nine patients (12.3\%). In 22 patients (30.1\%), the follow-up was longer than 5 years, and in five patients (6.8\%) it was longer than 10 years. The mean follow-up time was 35.6 months.

One month after the intervention, excellent results were achieved in 17 patients and good results, in 32 patients. Therefore, the short-term success rate was $67.1 \%$. Subsequent surgery at the index level was necessary in 19 patients (26.0\%). In these cases, the herniated disc fragment was removed, and a discectomy or a bony decompression of the spinal canal was performed.

Most reoperations (15 patients) had to be performed during the first year after PLDD (20.5\% of all patients, $78.9 \%$ of all resurgeries). These patients (Table 2) had a statistically significant worse outcome $(26.7 \%$ versus $75.0 \%$ satisfied patients, $|\mathrm{t}|=2.467,(\alpha 1=0.025) \mathrm{t}(7)=2.365)$. Radicular pain was present in all patients with an early subsequent surgery, but only in $50 \%$ of patients with late surgery $(\mathrm{P}=0.035)$. The mean time between PLDD and the additional surgery was at $10.8 \pm 17.9$ months (1-70 months).

Comparing the patients with excellent or good outcome (substantial pain relief) with the patients with poor pain relief (Table 3 ), significantly more patients with poor pain relief had radicular pain $(91.7 \%$ versus $69.4 \%, \mathrm{P}=0.04)$. As expected, the rate of resurgeries is higher if patients are not satisfied (50.0\% versus $14.3 \%, \mathrm{P}=0.002)$.

\section{Discussion}

This retrospective observational study investigated the number of patients with a subsequent open surgery after PLDD. Patients with back pain only and patients with radicular pain were included. The short-term success rate was $67.1 \%$; however, $26.0 \%$ of all patients had to undergo an additional surgery, most of them during the first year after PLDD. If resurgery was necessary, the primary outcome was worse compared to patients without surgery during follow-up. All patients with an early additional surgery had radicular pain. Patients with radicular pain had a worse outcome.

The short-term success rate is comparable with the few available studies from the literature. The recent study of McCormick et al. [6] found a 73\% positive response after one year using a threshold of $>50 \%$ improvement in NRS leg pain score and $>30 \%$ ODI improvement. This result builds on the other available studies with 6-to-24-month follow-up periods [9-13].

Also, the resurgery rate seems to be comparable with the only one study reporting these data. McCormick at al. [6] reported $36 \%$ additional surgery at the 8-year follow-up. All patients in his study had radicular pain. In the present study, the resurgery rate was $34 \%$ if only patients with radicular pain are taken into account. However, the patient selection in the McCormick study [6] and the present study is different. All patients in the McCormick study [6] were candidates for open discectomy. Therefore, it is concluded that PDLL had prevented spine surgery in $64 \%$ of cases. In the present study, no patient was a candidate for open surgery even though some of them had radicular pain or a sensory loss. However, no patient with a motor deficit or even bladder dysfunction was included. This means that the resurgery rate in the present study indicates additional surgery for a patient for whom conservative treatment was an alternative to PLDD. Recent studies found comparable resurgery rates for lumbar Nucleoplasty $(18.7 \%$, [16]) and for cervical Nucleoplasty (19.5 $\%$ [17]). 
TABLE 2: Patient characteristics dependent on the time of resurgery and significant differences between these two groups.

\begin{tabular}{|c|c|c|c|c|}
\hline & \multirow{2}{*}{ All Patients with Resurgery } & \multicolumn{2}{|c|}{ Resurgery } & \multirow{2}{*}{ Significance } \\
\hline & & during first year & later & \\
\hline $\mathrm{n}$ & 19 & $15(78.9 \%)$ & $4(21.1 \%)$ & \\
\hline Radicular pain & $17(89.5 \%)$ & $15(100.0 \%)$ & $2(50.0 \%)$ & $P=0.035$ \\
\hline \multicolumn{5}{|l|}{ Macnab's outcome criteria } \\
\hline mean & $2.9 \pm 0.8$ & $3.1 \pm 0.8$ & $2.3 \pm 0.5$ & $|t|=2.467,(\alpha 1=0.025) t(7)=2.365$ \\
\hline substantial pain relief $(1+2)$ & $7(36.8 \%)$ & $4(26,7 \%)$ & $3(75.0 \%)$ & \\
\hline \multicolumn{5}{|l|}{ Period until surgery (months) } \\
\hline mean & $10.8 \pm 17.9$ & $3.0 \pm 2.3$ & $40.3 \pm 20.8$ & $|t|=3.588,(\alpha 1=0.025) t(3)=3.182$ \\
\hline
\end{tabular}

TABLE 3: Patient characteristics dependent on the outcome and significant differences between these two groups.

\begin{tabular}{lcccc}
\hline & All Patients & Result & Significance \\
\hline $\mathrm{n}$ & 73 & $49(67.1 \%)$ & $24(32.9 \%)$ & \\
\hline Radicular pain & $56(76.7 \%)$ & $34(69.4 \%)$ & $22(91.7 \%)$ & \\
\hline Additional surgery & $19(26.0 \%)$ & $7(14.3 \%)$ & $12(50.0 \%)$ & $\mathbf{P . 0 4}$ \\
\hline
\end{tabular}

Avoidance of surgery is an important goal in reducing morbidity and mortality [6]. From the data of this study it remains unclear whether PLDD can achieve this objective. It is also worth considering whether radicular pain is a good indication for PLDD. Generally, patient selection appears to be extremely important in the efficacy of PDD $[8,18]$. The best results may be obtained when the disc herniation is contained $[8,18]$ and is limited to a single level [18]. For Ong et al. [8], the exact role of PLDD in the treatment of radicular pain is still up for debate, but PLDD should not be abandoned. Lee [19] concludes that, in spite of the lack of the evidence, the Dekompressor may be worth trying in patients with leg pain and contained disc herniations prior to open discectomy because the Dekompressor is easy to apply, is relatively safe, and causes less injurious to the disc. In contrast to the studies of Ong et al. [8] and Lee [19], the present study shows that radicular pain is an inferior indication compared to low back pain.

With an early resurgery, it was suspected that the indication for PLDD was too generous. Another explanation for early resurgery was the risk of acute herniation after the puncture of the disc with the 17-gauge needle. The risk of acute herniation is dependent on the needle diameter. The most vulnerable site is the inner annulus [20]. All patients with an early additional surgery had radicular pain. Patients with radicular pain had worse outcomes compared to patients with back pain only. As a consequence, a good indication might be a patient with low back pain without radicular symptoms with a contained disc. Trying Dekompressor in the first instance risks an additional surgery with lower success rates [1, 21]. This study suggests that Dekompressor is unable to replace open surgery.

There are limitations to this study. This audit is retrospective and observational and therefore does not represent a high level of evidence. However, the resurgery rate is an important factor for the outcome.

\section{Conclusions}

At first sight, a satisfied patient level of $67 \%$ seems to be a good result. However, this short-term result is significantly worsened due to a resurgery rate of $26.0 \%$. Subsequent surgery a short time after PLDD suggests that PLDD is not a replacement for open discectomy. A contained disc herniation causing low back pain without radicular pain appears to be a good indication for Dekompressor. Because patients with radicular pain had a worse outcome and more frequent resurgeries, whether radicular pain is an ideal indication for PDLL should be discussed. Further studies are needed to compare the outcome and rate of subsequent surgery in patient populations with and without radicular symptoms to find the ideal indications for PLDD.

\section{Data Availability}

The data used to support the findings of this study are available from the corresponding author upon request.

\section{Conflicts of Interest}

The author declares that there are no conflicts of interest regarding the publication of this paper.

\section{References}

[1] Y. Vorobeychik, V. Gordin, D. Fuzaylov, and M. Kurowski, "Percutaneous Mechanical Disc Decompression Using Dekompressor Device: An Appraisal of the Current Literature," Pain Medicine, vol. 13, no. 5, pp. 640-646, 2012.

[2] H. Weber, "Lumbar disc herniation: a controlled, prospective study with ten years of observation," The Spine Journal, vol. 8, no. 2, pp. 131-140, 1983.

[3] J. N. Weinstein, T. D. Tosteson, J. D. Lurie et al., "Surgical vs nonoperative treatment for lumbar disk herniation. The Spine 
Patient Outcomes Research Trial (SPORT): A randomized trial," Journal of the American Medical Association, vol. 296, no. 20, pp. 2441-2450, 2006.

[4] J. N. Weinstein, J. D. Lurie, T. D. Tosteson et al., "Surgical versus nonoperative treatment for lumbar disc herniation: four-year results for the Spine Patient Outcomes Research Trial (SPORT)," The Spine Journal, vol. 33, no. 25, pp. 2789-2800, 2008.

[5] S. J. Atlas, R. B. Keller, Y. A. Wu, R. A. Deyo, and D. E. Singer, "Long-term outcomes of surgical and nonsurgical management of sciatica secondary to a lumbar disc herniation: 10 year results from the Maine lumbar spine study," The Spine Journal, vol. 30, no. 8, pp. 927-935, 2005.

[6] Z. L. McCormick, C. Slipman, A. Kotcharian et al., "Percutaneous lumbar disc decompression using the dekompressor: A prospective long-term outcome study," Pain Medicine, vol. 17, no. 6, pp. 1023-1030, 2016.

[7] V. Singh, R. M. Benyamin, S. Datta, F. J. Falco, S. Helm II, and L. Manchikanti, "Systematic review of percutaneous lumbar mechanical disc decompression utilizing Dekompressor," Pain Physician, vol. 12, no. 3, pp. 589-599, 2009.

[8] D. Ong, N. H. L. Chua, and K. Vissers, "Percutaneous Disc Decompression for Lumbar Radicular Pain: A Review Article," Pain Practice, vol. 16, no. 1, pp. 111-126, 2016.

[9] D. Erginousakis, D. K. Filippiadis, A. Malagari et al., "Comparative prospective randomized study comparing conservative treatment and percutaneous disk decompression for treatment of intervertebral disk herniation," Radiology, vol. 260, no. 2, pp. 487-493, 2011.

[10] J. Aronsohn, K. Chapman, and M. Soliman, "Percutaneous microdiscectomy versus epidural injection for management of chronic spinal pain," Proc West Pharmacol Soc, pp. 16-19, 2010.

[11] K. M. Alò, R. E. Wright, J. Sutcliffe, and S. A. Brandt, "Percutaneous lumbar discectomy: One-year follow-up in an initial cohort of fifty consecutive patients with chronic radicular pain," Pain Practice, vol. 5, no. 2, pp. 116-124, 2005.

[12] P. Lierz, K. M. Alo, and P. Felleiter, "Percutaneous lumbar discectomy using the dekompressor ${ }^{\circledR}$ system under CT-control," Pain Practice, vol. 9, no. 3, pp. 216-220, 2009.

[13] J. Lemcke, F. Al-Zain, S. Mutze, and U. Meier, "Minimally invasive spinal surgery using nucleoplasty and the dekompressor tool: A comparison of two methods in a one year follow-up," Minimally Invasive Neurosurgery, vol. 53, no. 5-6, pp. 236-242, 2010.

[14] R. W. Abdu, W. A. Abdu, A. M. Pearson, W. Zhao, J. D. Lurie, and J. N. Weinstein, "Reoperation for Recurrent Intervertebral Disc Herniation in the Spine Patient Outcomes Research Trial," The Spine Journal, vol. 42, no. 14, pp. 1106-1114, 2017.

[15] C. B. Dewing, M. T. Provencher, R. H. Riffenburgh, S. Kerr, and R. E. Manos, "The outcomes of lumbar microdiscectomy in a young, active population: Correlation by herniation type and level," The Spine Journal, vol. 33, no. 1, pp. 33-38, 2008.

[16] S. Klessinger, "The frequency of re-surgery after lumbar disc Nucleoplasty in a ten-year period," Clinical Neurology and Neurosurgery, vol. 170, pp. 79-83, 2018.

[17] S. Klessinger, "The frequency of re-surgery after cervical disc nucleoplasty," World Neurosurgery, vol. 117, pp. e552-e556, 2018.

[18] L. Manchikanti, V. Singh, A. K. Calodney et al., "Percutaneous lumbar mechanical disc decompression utilizing Dekompressor ${ }^{\circledR}$ : an update of current evidence.," Pain Physician, vol. 16, no. 2, p. $-24,2013$.
[19] S. C. Lee, "Percutaneous intradiscal treatments for discogenic pain," Acta Anaesthesiologica Taiwanica, vol. 50, no. 1, pp. 2528, 2012.

[20] V. M. van Heeswijk, A. Thambyah, P. A. Robertson, and N. D. Broom, "Does an Annular Puncture Influence the Herniation Path?” An In Vitro Mechanical and Structural Investigation, 2017.

[21] A. Vik, J. A. Zwart, G. Hulleberg, and P. Nygaard, "Eight year outcome after surgery for lumbar disc herniation: A comparison of reoperated and not reoperated patients," Acta Neurochirurgica, vol. 143, no. 6, pp. 607-611, 2001. 


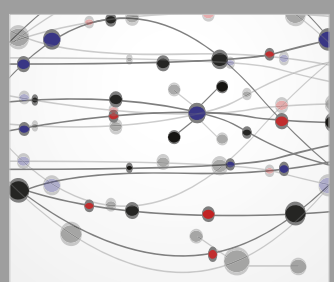

The Scientific World Journal
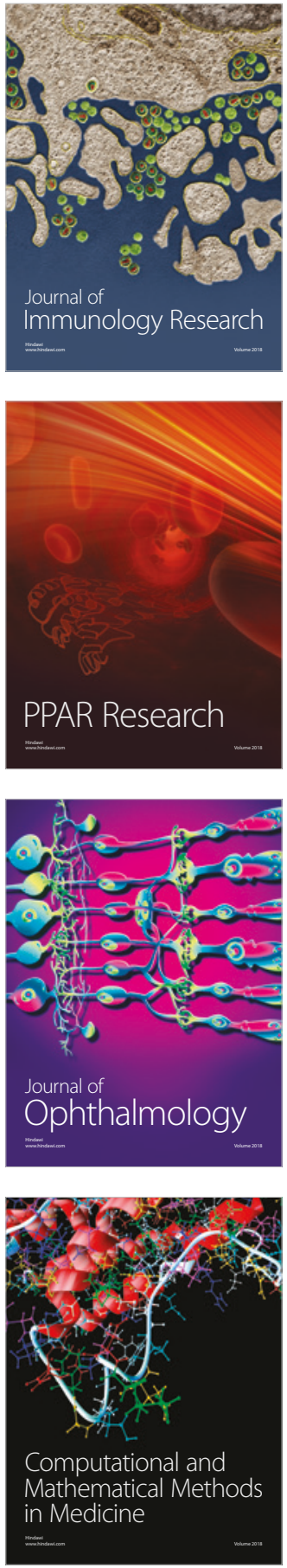

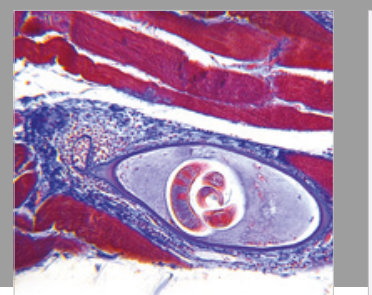

Gastroenterology Research and Practice

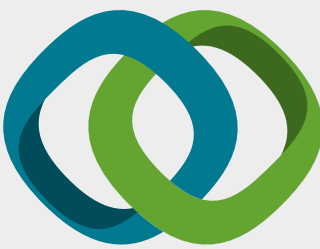

\section{Hindawi}

Submit your manuscripts at

www.hindawi.com
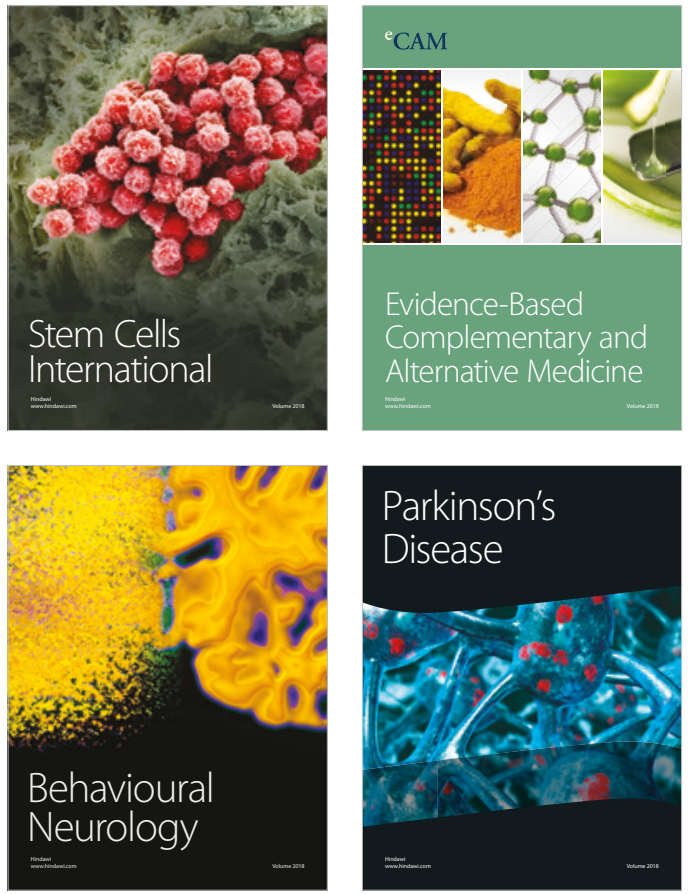

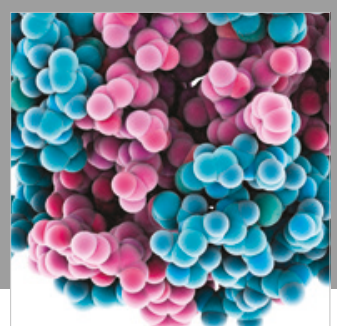

ournal of

Diabetes Research

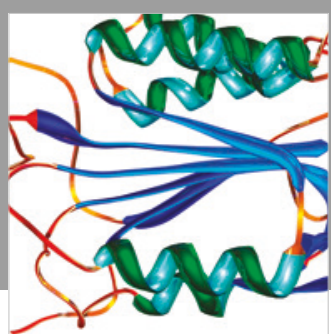

Disease Markers
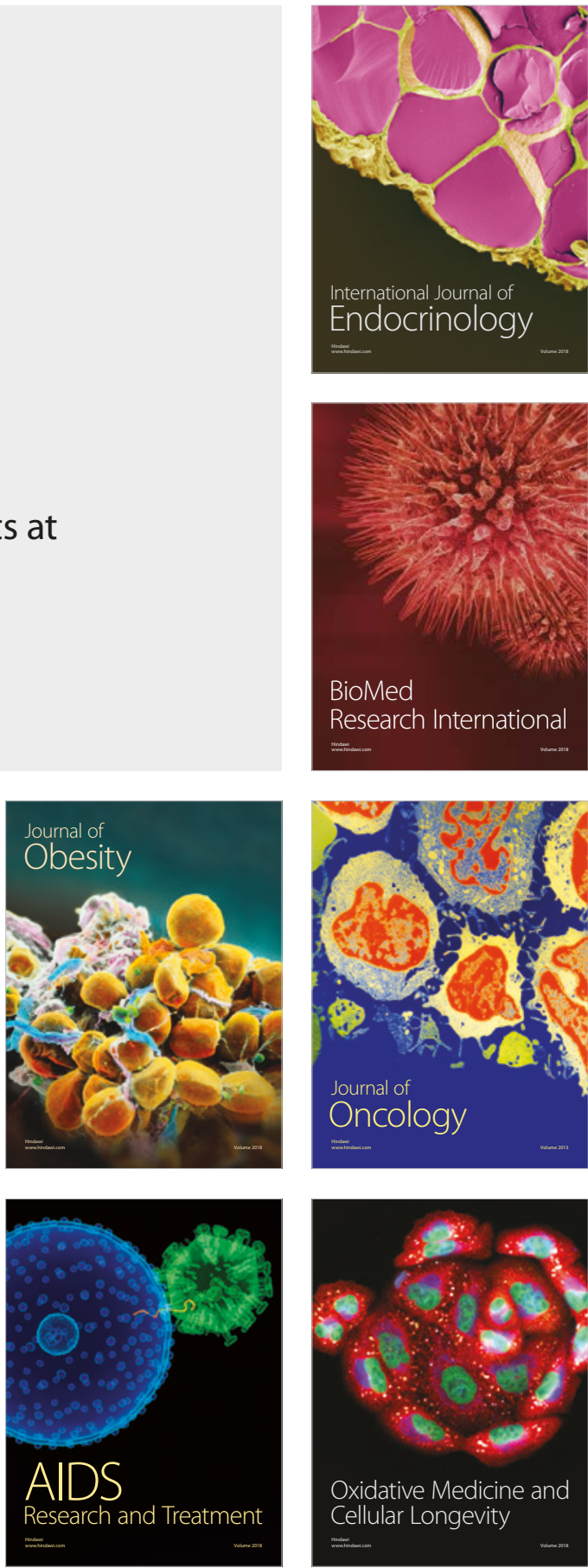